invasion. My own observations would support this, for, as I have stated above, the more active the lesion the more extracellular were the organisms. On the other hand, I think that in many cases a mixed infection with other pyogenic germs plays an important part. The gonococcus is rarely isolated in pure culture and if found at all it is usually only during the earlier stages. It would be surprising if mixed infection did not occur, considering the ease with which pyogenic germs could invade the body through the primary gonorrhoeal lesions. The bacillus coli seems to be the organism most usually found, which is in correspondence with the fact that it is the commonest organism found in cystitis and other diseases of the urinary passages. When found in lesions after death little importance can be placed in its presence as it is found so often in a variety of diseases from "agonal" or post-mortem invasion. But whatever organism be found in association with the gonococcus its action is, I believe, purely secondary. It completes the work begun by the gonococcus which being of more delicate growth disappears before its stronger competitors.

Diagnosis.-Cases of gonorrhœeal pyæmia and septicæmia are rarely diagnosed during life. This is partly due to the fact that very little attention has been paid to the possible gonorrhoeal origin of pyæmia, but also because these cases do not often occur in connexion with an acute gonorrhcea but from secondary foci in the prostate or seminal vesicles which are often latent. Gonorrhcea may be clenied by the patient and the chronic gleety discharge overlooked by his attendant. Ullmann ${ }^{11}$ reported five sases of fatal gonorrhœal pyæmia, in four of which the necropsy showed the point of origin to be a prostatic abscess. In three of the cases the clinical diagnoses were typhoid fever, malignant tumour of the prostate, and purulent cystitis and septicæmia of doubtful origin. Such cases show the need for the routine examination of the urinary organs and especially of the prostate in all cases of pyæmia of obscure causation, just as the middle ear is now always examined in similar cases. Gonorrbcal infection has no special clinical features which differentiate it from other pyæmias and a positive diagnosis can only be made by a bacteriological examination of the blocd or of any accessible lesions.

Technique.-For the examination of the blood stained films are not sufficient; cultures must be made upon suitable media. The essential constituent of these media seems to be blood serum or similar albuminous material from the animal body. Practically blood agar is the most convenient means of cultivating the gonococcus. At least one cubic centimetre of blood should be taken with a sterilised hypodermic syringe from one of the large veins of the elbow. No antiseptic must be used in sterilising the skin; simple cleansing with spirit and ether is sufficient for this purpose. The blood should then be poured on to the surface of one or two agar "slopes," the tubes being kept slightly inclined until the blood has coagulated. A relatively large quantity of blood must be used as it is necessary to have, as Harris ${ }^{\text {in }}$ has shown, abundant albuminous material present. Another method is to mix the blood with one or two tubes of melted agar kept at a temperature of $40^{\circ} \mathrm{C}$., the mixture then being slanted or plated in a Petri's dish. The former method I think is preferable, as a freer access of oxygen is obtained than by mixing the blood with the agar. I have failed with the latter method when the former has been quite successful. For the same reason fluid media are unsuitable as the blood clots and falls to the bottom. For cultivation from pus or lesions of the organs blood agar can also be used. The blood must be liberally distributed over the surface of agar "slants" and not simply a few drops smeared over it. Serum agar is also an excellent medium, one part of blood serum being used to two or three parts of agar. Young ${ }^{13}$ has used "hydrocele fluid" successfully, while Wright ${ }^{14}$ advocates " urine-serum-agar," prepared by mixing under aseptic precautions with melted agar at a temperature of $40^{\circ}$, one-third to one-half of its volume of a sterile mixture of beef-blood serum (two parts) and human urine (one part). I have had no personal experience of this last method. The identification of the gonococcus is usually said to depend upon three characteristics: (1) characteristic colonies are produced upon certain media; (2) it will not

11 Ullmann: Deutsches Archiv fur Klinische Medicin, 1901, Band lxix. S. 309 .

12 Harris : Bulletin of the Johns Hopkins Hospital, October, 1902. 13 Young . Journal of Cutaneous and Genito-Urinary Diseases, June, 1900 .

14 Wright : American Journal of the Medical Sciences, January, 1895 grow upon simple agar or in broth; and (3) it is not stained by Gram's method. Of these, only the first is certain. The colonies appear after 24 hours as minute, greyish, semitranslucent points. In 48 hours they are well developed and under a low magnifying power each is seen to be circular with thin, translucent, sharply defined margins. The margins are slightly undulated and in older colonies may send out slight processes. The colonies become brownish, denser, and granular towards the centre. Wildbolz ${ }^{15}$ has succeeded in growing the gonococcus upon agar after cultivating on serum agar for four or five generations, sometimes earlier. This test cannot therefore be relied upon especially as the diplococcus meningitidis is also difficult to grow upon agar. 'There are also fallacies in the test by Gram's method. Colonies not more than 48 hours old must be used, as other cocci usually staining by Gram's method may be decolourised in older cultures. Mallory and Wright ${ }^{16}$ found a Gram-decolourising coccus in an arthritis of the knee, clinically of gonorrheal origin, which in films was regarded as the gonococcus, but cultures proved that it was not that organism. In staining suspected films by Gram's method it is useful to place a few staphylococci in one corner of the slide for a comparison. Practically, then, most reliance must be placed on the appearance of the colonies grown on blood or serum agar and if these colonies are composed of cocci of characteristic appearance and decolourised by Gram's method the identification is certain.

When in cases of obscure pyæmia the blood has been examined during life with negative results I would suggest that the blood from the heart and spleen should be examined as soon as possible after death. Permission for this could often be obtained when an ordinary post-mortem examination would not be allowed. It is also useful when a necropsy is obtained, as bacteriological examinations of the organs and blood 24 hours after death are made untrustworthy by the invasion of other organisms, especially from the intestines.

I must, in conclusion, express my indebtedness to Mr. Barling and Dr. Simon for their kind permission to make use of their cases.

Birmingham.

\section{A HAT PIN +3 INCHES LONG IN THE ESOPHAGUS PRODUCING A FALSE ANEURYSM OF THE SUPERIOR MESENTERIC ARTERY.}

BY H. D. ROLLESTON, M.D. CANTAB., F.R.C.P. LOND., PHYSICIAN TO ST. (IEORGE'S HOSPITAL; AND

T. R. C. WHIPHAM, M.B. OxON, M.R.C.P. Lond.,

ASSISTANT CLRATOR OF THE MUSEUM AT ST. GEORGE'S HOSPITAL; PHYSICLAN TO OUT-PATIENTS AT 'LHE EVELINA HOSPITAL FOR SICK CHIXDREN.

A FEMALE child, three years of age, who had been ailing for two months, was admitted into St. George's Hospital on June 25th, 1904, with wasting, anæmia, rickets, inability to sit up, and a distended abdomen. A diagnosis of tuberculous peritonitis was made and on July 6th the patient was transferred for further treatment to the Atkinson Morley Convalescent Hospital at Wimbledon. While there the child did not improve; she lost weight, developed retraction of the head, and on August 18th vomited some fresh blood clot of the size of a grape and on two subsequent occasions brought up some dark, discoloured blood. She was re-admitted to St. George's Hospital on August 24th, looking like a most advanced case of tuberculous peritonitis, and she continued to get worse. On the 30th she vomited a clot of fresh blood of about the size of a walnut and, becoming very collapsed, died at 8 P.M.

The diagnosis of tuberculous peritonitis rested on the swollen abdomen, the diarrhoea, the enlargement of the spleen and liver, and the wasting; while crackles at the apices of the lungs and retraction of the head suggested more widespread tuberculosis. Hæmatemesis in tuberculous

15 Wildholz: Centralblatt frir Bakteriologie, Feb. 14th, 1902 ; and Brit. Merl. Jour., Eipitome, May 31st, 1902. 16 Mallory and Wright: Pathological Technique, p. 146 
peritonitis is such an unusual event that a necropsy was highly desirable, as it was thought that it might disclose some rare condition, such as tuberculous ulceration of the stomach or of the cesophagus.

Necropsy. - The body was thin and showed marked signs of rickets. There was no external wound or lesion of the skin except some small quite superficial scars over the sacrum, probably the result of pressure sores. The oesophagus (Fig. 1) contained part of a long black pin, resembling a hat pin, of which two and a half inches of the pointed end projected upwards through a small hole in the wall of the gullet on the right side just above the level

FIG. 1.

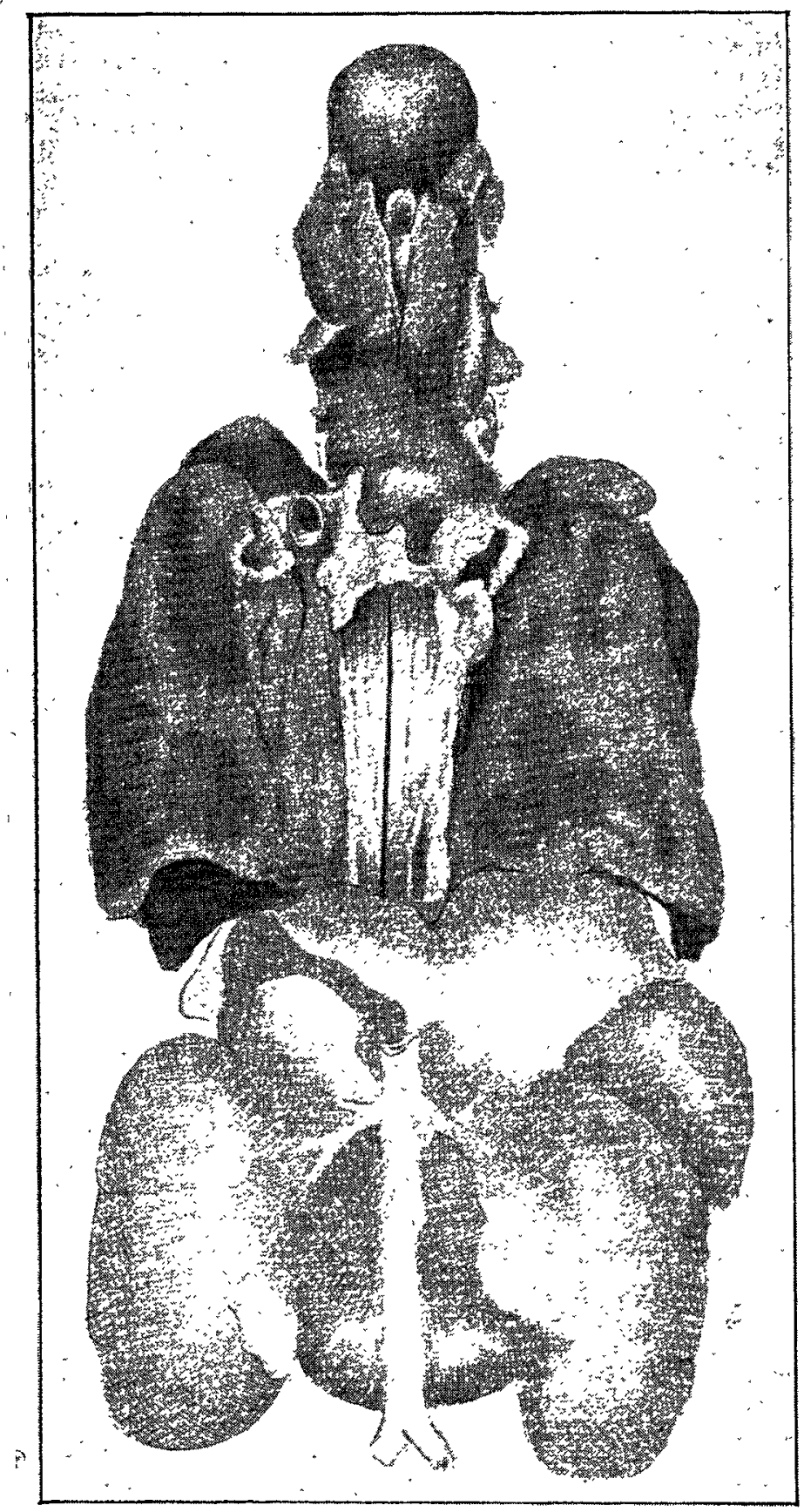

CEsophagus opened from the back showing the sharp pointed end of the hat pin pointing upwards from the spot where it penetrated the osophageal wall. To the right of the cesophagus in this situation a suppurating $\mathrm{g}^{\prime}$ and is seen.

of the diaphragm. The pin, which was four and threequarter inches long and partially eroded, had evidently been swallowed head downwards. The actual head of the pin, which was missing and could not be found, had probably become detached soon after swallowing and had been voided per vias naturales. The shaft was slightly bent with the concavity forwards and the head end. which was very sharp but not shaped to a point like the other, had penetrated the oesophageal wall at the point described and passing in front of the aorta had pierced the diaphragm and had travelled downwards behind the stomach without injuring it through the extreme posterior portion of the pancreas; thence it turned a little forwards to a point immediately above the duodenum where it had perforated the upper wall of the superior mesenteric artery (Fig. 2). Between the pancreas and the duodenum there was a small false aneurysm filled with blood clot and of the size of a cherry in the root of the mesentery. The track of the pin above this was also filled with blood. The superior mesenteric vein, which lay to the right and slightly in front of the aneurysm, was partially thrombosed with ante-mortem clot. The point or upper end of the pin lay in the cesophagus opposite the apices of the lower lobes of the lungs posteriorly. At this level there was a small suppurating lymphatic gland

FrG. 2.

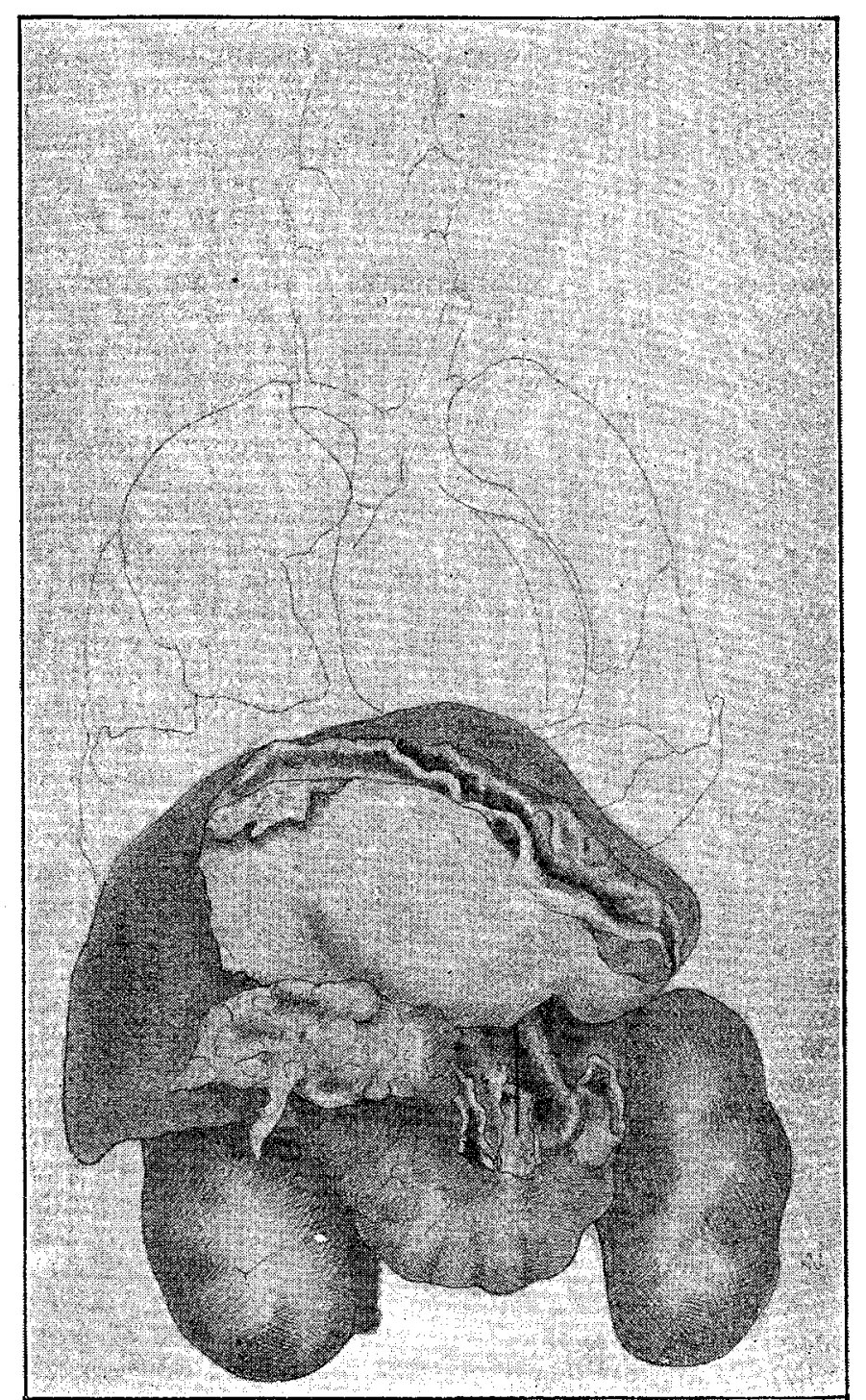

Shows the end of the pin penetrating the superior mesenteric artery.

behind the osophagus with a small abscess on either side of it; that on the left was the larger of the two and had invaded to a slight extent the lower lobe of the lung, causing several small foci of suppuration around. A direct communication was found between the cesophagus and the abscess on the right and a similar condition had possibly existed on the left side. Unfortunately in removing the contents of the thorax the cesophagus was toin in this position, so that this point could not be established; at all events the wall was much softened and very friable. From the abscess cavity on the right a sinus, large enough to admit the extreme tip of the little finger, passed upwards to the first dorsal vertebra. This sinus, which was apparently of some little standing, contained small flakes of necrosed bone. The body of the first dorsal vertebra was slightly necrosed and the bodies of the next four dorsal vertebræ were extensively carious, those of the third, fourth, and fifth having almost entirely disappeared; the process stopped at the upper part of the body of the sixth. The second to the sixth costal articulations on the right side and the third to the sixth on the left were also extensively eroded. There was recent fibrinous pleurisy orer the posterior 
surfaces of both lungs with recent adhesions on the right. The lower lobe of the right lung showed some collapse. No sign of tubercle was visible in either. The pericardium contained an excess of serous fluid. The heart and other viscera were normal and presented no evidence of tuberculosis. The stomach was filled with blood clot and the intestines contained a considerable quantity. There was no ulceration or other lesion of the stomach or intestines, so that it seems probable that the blood had tracked up from the aneurysm along the course of the pin and entered the alimentary canal at the perforation of the csophagus. There was no sign of injury, either recent or old, on the palate or in the pharynx. The brain was normal and there was no meningitis.

The lesions of the spinal column gave every appearance of being of long standing and tuberculous in origin and the infiltration of the left lung from the suppurating gland and the sinus on the right side suggested that the process had not occurred merely a short time before death, as must have happened if the condition was due solely to perforation by the point of the pin. The clinical history that blood had only been vomited during the last two weeks and the fact that only the upper wall of the superior mesenteric artery had been pierced indicate that the pin had only recently reached the position in which it was found post mortem. Before this it must have been above the level of the suppuration and therefore not the cause of it. Tuberculous osteitis of the dorsal spine and the suppuration in the adjacent mediastinal gland were therefore probably antecedent to, and independent of, the perforation of the neighbouring part of the esophagus by the pin. In all probability the point of the pin perforated the wall of the cesophagus, softened by suppuration from the spine, shortly before death.

As has been pointed out, the presence of the pin in the œsophagus was not suspected during life. It, however, appeared at the inquest that the mother believed that the child had swallowed a pin and indeed had mentioned her belief at another hospital, but not meeting with much sympathy there had refrained from saying anything about it at St. George's Hospital. The pin was evidently swallowed head downwards and its rounded end and the flexibility of its shaft must have enabled it to glide along the posterior wall of the pharynx into the cesophagus. In a very similar case recorded by Holding ${ }^{1}$ a female child aged 20 months probably fell on the head-end of a hat pin which passed into the stomach and after being localised by skiagraphy was successfully removed by gastrostomy. It is noteworthy that in our case the pin had been in the cesophagus for over two months.

It may be added that during the last few years there have been at least ten other cases of foreign bodies, other than food, impacted in the csophagus at St. George's Hospitalviz., four cases of tooth plates, of which three terminated fatally, one case of a pin which perforated the aorta and proved fatal (recorded by Sir Isambard Owen), ${ }^{2}$ three cases of halfpennies, one case of a whistle, and one of a brooch, all swallowed by children and all treated successfully. For the two illustrations we are indebted to the skill of Mr. Lawrence Jones.

\section{MANDIBULAR PROCESSES ASSOCIATED WITH DOUBLE HARE-LIP AND CLEFT PALATE.}

\section{BY WALIER LEY WOOLLCOMBE, M.R.C.S. ENG.,} F.R.C.S. EDIN.,

SURGEON TO THE SOUTH DEYON AYD EAST CORNWALL MOSPITAL.

T'wo children have recently been under my care for double hare-lip and cleft palate both of whom exhibited a peculiar deformity of the lower lip, which has been described and figured by Mr.J. Bland-Sutton ${ }^{3}$ and others ${ }^{4}$ and attributed by them to imperfect coalescence of the mandibular processes, but which I have never seen before in 18 years of hospital practice nor do many others seem to have described it.

1 Annals of Surgery, Part cxli., September, 1904, p. 354. Brit. Med. Jour, vol. i., 1903 , p. 1490

3 Tumours, Innocent and Malignant, 1901 edition, p. 362

4 Transactions of the Clinical Society, vol. xxiv., p. 230 ; Langenbeck" Archives, band xxxvii., p. 230 .
CASE 1. - The patient was a boy, aged three months (sent by Mr. A. E. Gladstone of Newton Ferrers), whose father was born with hare-lip and cleft palate but who had two sisters without this deformity. The father and mother were not related. The boy had a complete cleft of the hard and soft palate and the worst looking hare-lip I have ever seen owing to the fact that the clefts on either side of the premaxilla were very wide and that the latter process was held forward by the vomer so that it stood out three-quarters of an inch in front of the rest of the upper lip. On the lower lip, which was perfect in outline, and at a distance of one centimetre on either side of the mid-line, were two depressions out of each of which protruded a nipple-shaped process about one centimetre in length which is diagrammatically represented in Fig. 1 . These processes were capable of

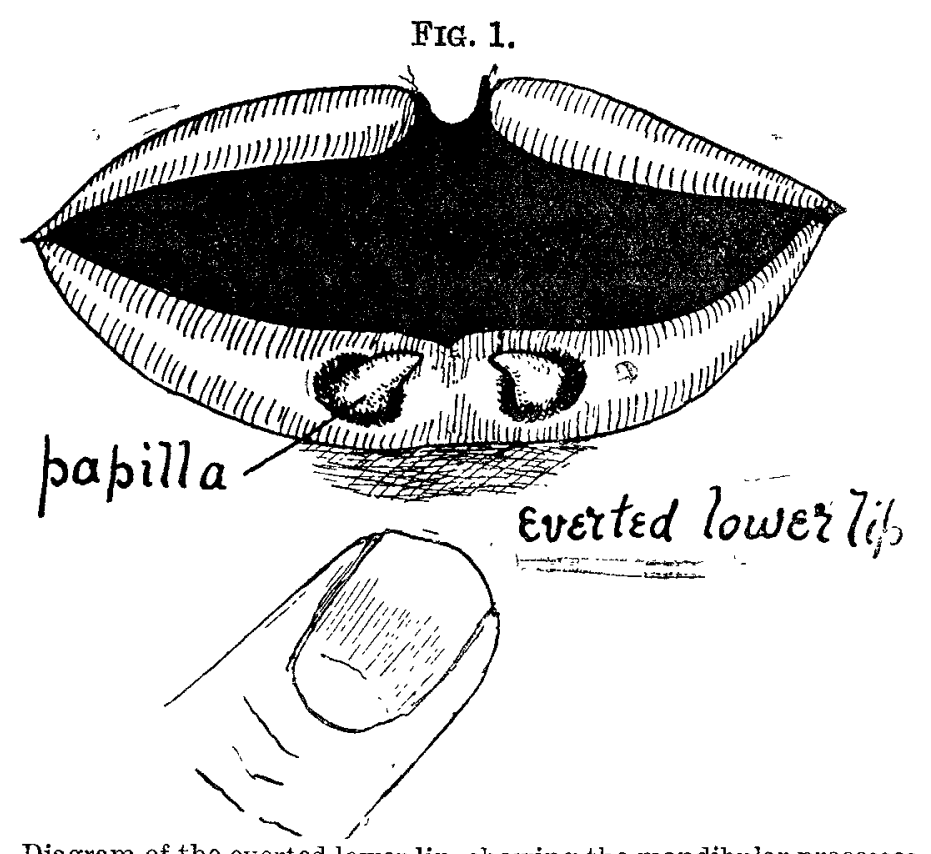
and $\operatorname{lip}$, showing

a good deal of movement and several times per minute were protruded and retracted in a most uncanny way like feelers. The recesses were always full of mucus. The upper lip was treated by peeling back the periosteum from, and excising three-quarters of an inch of the central part of, the vomer, pressing the premaxilla back into the interval between the separated alveolar borders of the superior maxillæ, suturing it in place after freshening the opposing surfaces, then uniting flaps from the lips on either side, utilising the soft parts covering the premaxilla to fill in the central gap. The lower lip was subsequently treated by excising an elliptical portion of it, including the two recesses and tubercles, going deeply enough to remove all the glandular tissue connected with them.

CASE 2.-The patient was a boy, aged four months (sent by Mr. E. O. Kingdon of Holsworthy), who came of a healtby stock and had healthy brothers, no other member of the family showing any deformity. The condition was exactly similar to that in Case 1, except that the recesses and tubercles were much smaller and did not secrete nearly so much mucus.

The facts that these recesses and processes have never been observed apart from double hare-lip-i.e., imperfect coalescence of the naso-frontal and maxillary processes-and also that Feurer once found a similar recess in the upper lip at the junction of these processes make it appear likely that the deformity is in some way connected with the intermandibular fissure, but this reasoning does not seem to me to explain satisfactorily the following points: (1) There are always a pair of recesses and they always seem to be situated at an equal and definite distance from the mid-line (about one centimetre); (2) there is never any irregularity in the line of the lip, which is perfectly united at the mid-line; and (3) the large amount of glandular tissue grouced around the recess.

The excised part of the lower lip bearing the two papilla $r$ processes was submitted for examination to Dr. Arthur Keith who reported as follows: "Each process is made of three elements: (1) a clump of mucous glands which forms the core : the ducts of these glands open close together near the apex of the process; (2) a layer of muscular tissue continuous with the orbicularis oris which surrounds the 